\title{
The white dwarf population of NGC 6397
}

\author{
Santiago Torres ${ }^{1,2}$, Enrique García-Berro ${ }^{1,2}$, Leandro G. Althaus ${ }^{3,4}$, and María E. Camisassa ${ }^{3,4}$ \\ ${ }^{1}$ Departament de Física Aplicada, Universitat Politècnica de Catalunya, c/Esteve Terrades 5, 08860 Castelldefels, Spain \\ e-mail: enrique.garcia-berro@upc.edu \\ 2 Institute for Space Studies of Catalonia, c/Gran Capita 2-4, Edif. Nexus 104, 08034 Barcelona, Spain \\ 3 Facultad de Ciencias Astronómicas y Geofísicas, Universidad Nacional de La Plata, Paseo del Bosque s/n, 1900 La Plata, Argentina \\ ${ }^{4}$ Instituto de Astrofísica de La Plata, UNLP-CONICET, Paseo del Bosque s/n, 1900 La Plata, Argentina
}

Received 22 March 2015 / Accepted 31 July 2015

\section{ABSTRACT}

\begin{abstract}
Context. NGC 6397 is one of the most interesting, well-observed, and most thoroughly theoretically studied globular clusters. The existing wealth of observations allows us to study the reliability of the theoretical white dwarf cooling sequences of low-metallicity progenitors, to determine the age of NGC 6397 and the percentage of unresolved binaries. We also assess other important characteristics of the cluster, such as the slope of the initial mass function or the fraction of white dwarfs with hydrogen-deficient atmospheres. Aims. We present a population synthesis study of the white dwarf population of NGC 6397. In particular, we study the shape of the color-magnitude diagram and the corresponding magnitude and color distributions.

Methods. To do this, we used an advanced Monte Carlo code that incorporates the most recent and reliable cooling sequences and an accurate modeling of the observational biases.

Results. Our theoretical models and the observed data agree well. In particular, we find that this agreement is best for those cooling sequences that take into account residual hydrogen burning. This result has important consequences for the evolution of progenitor stars during the thermally pulsing asymptotic giant branch phase, since it implies that appreciable third dredge-up in low-mass, lowmetallicity progenitors is not expected to occur. Using a standard burst duration of $1.0 \mathrm{Gyr}$, we obtain that the age of the cluster is $12.8_{-0.75}^{+0.50}$ Gyr. Greater ages are also compatible with the observed data, but then unrealistic longer durations of the initial burst of star formation are needed to fit the luminosity function.

Conclusions. We conclude that a correct modeling of the white dwarf population of globular clusters, used in combination with the number counts of main-sequence stars, provides a unique tool for modeling the properties of globular clusters.
\end{abstract}

Key words. globular clusters: individual: NGC 6397 - stars: luminosity function, mass function - globular clusters: general white dwarfs

\section{Introduction}

White dwarfs are the remnants of the evolution of single stars of low and intermediate mass. Consequently, they gather important information not only about the evolution of their progenitor stars, but also about the properties of their parent populations. Moreover, since in general terms the evolution of white dwarf stars is relatively well understood, the ensemble properties of their populations can be used to test the validity of physical theories, which cannot be done using terrestrial facilities (García-Berro et al. 1995, 2011a; Isern et al. 1992, 2008, 2010; Córsico et al. 2012). This has been possible because we now have a profound knowledge of the physics governing white dwarf interiors and envelopes, which has resulted in accurate cooling tracks, as well as a wealth of observational data. Even more, the quality of the available observational data has allowed us to check the accuracy of our description of the main physical processes involved in their evolution - see, for instance, Althaus et al. (2010) for a recent review. In this vein, it is important to realize that because white dwarf populations of globular clusters are conceptually simple, their properties can be used to test white dwarf evolutionary models. This is particularly interesting because it has recently been shown that under controlled conditions, theoretical white dwarf cooling times obtained using very different numerical codes and techniques do not differ by much. Hence, white dwarf evolutionary times should be at least as reliable as main-sequence lifetimes (Salaris et al. 2013).
Several studies have taken advantage of all these properties and have determined accurate ages of both open and globular clusters. For instance, García-Berro et al. (2010) determined the age of the old, metal-rich, well-populated, open cluster NGC 6791 using the termination of the cooling sequence (Bedin et al. 2005, 2008a,b), and solving the long-standing problem of the discrepancy between the main-sequence and white dwarf ages. But this is not the only cluster for which we have good age determinations using white dwarf evolutionary sequences. Other examples are the young open clusters M 67 (Bellini et al. 2010), NGC 2158 (Bedin et al. 2010), and NGC 6819 (Bedin et al. 2015), the globular clusters M 4 (Hansen et al. 2002; Bedin et al. 2009), $\omega$ Centauri (Bellini et al. 2013), and NGC 6397 (Hansen et al. 2013), or the metal-rich globular cluster 47 Tuc (Goldsbury et al. 2012; García-Berro et al. 2014).

NGC 6397 is the second-nearest globular cluster to the Sun and has been thoroughly observed by the Hubble Space Telescope. Thus, we have high-quality deep images for it that have allowed us to study not only the lower main sequence, but also the white dwarf cooling sequence. NGC 6397 is old and metal-poor, its metallicity is $[\mathrm{Fe} / \mathrm{H}]=-1.8$ (Hansen et al. 2013), although there exists some discrepancy about its precise value (Richer et al. 2008) in the recent literature. The same is true for the age of the cluster. For instance, Hansen et al. (2007) analyzed the position of the cutoff of the white dwarf luminosity function and, comparing this with theoretical cooling models, derived an 
age $T_{\mathrm{c}}=11.47 \pm 0.47$ Gyr. Similarly, Winget et al. (2009) simultaneously fitted the main-sequence, the pre-white dwarf, and the white dwarf regions of the color-magnitude diagram, and obtained an age $T_{\mathrm{c}}=12.0_{-1.0}^{+0.5} \mathrm{Gyr}$. These ages, which are based on white dwarf evolutionary models, need to be compared with those obtained by fitting the luminosity of the main-sequence turn-off (MSTO) - see Richer et al. (2008) for a discussion of different methods and age estimates of NGC 6397. In particular, it is worth highlighting that using this method, Gratton et al. (2003) derived an age of $13.9 \pm 1.1 \mathrm{Gyr}$. This prompted Strickler et al. (2009) to claim that this age determination was compatible with the possible existence of a putative population of very old helium white dwarfs. On the other hand, AnthonyTwarog \& Twarog (2000) obtained 12.0 \pm 0.8 Gyr and Chaboyer et al. (2001) derived $13.4 \pm 0.8$ Gyr. Thus, the precise age of NGC 6397 remains unclear and needs to be independently evaluated using the most recent white dwarf evolutionary tracks of the appropriate metallicity. To this we add that NGC 6397 shares some other interesting characteristics with other Galactic globular clusters, such as the unusual lithium enhancement of some of their stars - see Pasquini et al. (2014) and references therein or the possible existence of multiple populations (di Criscienzo et al. 2010; Milone et al. 2012). Thus, deriving an independent white dwarf cooling age for this globular cluster is of crucial importance.

Moreover, NGC 6397 is one of the few globular cluster for which, in addition to its color-magnitude diagram, we also have an accurate white dwarf luminosity function, thus allowing us to investigate in detail the issues mentioned above. Specifically, Winget et al. (2009) used the shape of the white dwarf luminosity function of NGC 6397 to constrain important properties of white dwarf interiors. They found that to account for the observed shape of the white dwarf luminosity function of NGC 6397, either a low oxygen mass fraction in the inner carbon-oxygen core of typical white dwarfs was needed, or the crystallization temperature of the carbon-oxygen dense binary plasma would need to be significantly higher than that of a one-component plasma. The reason for this is that when the carbon-oxygen plasma crystallizes, the oxygen abundance in the solid phase is enhanced (Garcia-Berro et al. 1988a,b; Horowitz et al. 2010). This leads to a redistribution of oxygen in the core of the white dwarf because the inner regions are enriched in oxygen, while the outer ones become oxygen-poor. Since oxygen is slightly heavier than carbon, this chemical separation process releases gravitational energy, increasing the cooling times (Isern et al. 1997, 2000). Winget et al. (2009) found that to fit the observations, the effects of phase separation upon crystallization should be minimized. Hence, either the gravitational energy should be released at higher luminosities (or, equivalently, core temperatures), or the oxygen abundance in the deep interior of typical white dwarfs would need to be lower. This, in turn, is an important topic, as a low oxygen mass fraction could be indicative of a small cross section of the poorly measured $\mathrm{C}^{12}(\alpha, \gamma) \mathrm{O}^{16}$ nuclear reaction, since it has implications for the evolution of white dwarf progenitors (Salaris et al. 1997). Moreover, since the precise value of the $\mathrm{C}^{12}(\alpha, \gamma) \mathrm{O}^{16}$ cross section is still the subject of an active debate - see, for instance, Avila et al. (2015), and references therein - any additional piece of evidence helping in constraining it is valuable.

Furthermore, recent theoretical models predict that at moderately low luminosities a phase of stable hydrogen nuclear burning in a shell ensues in the atmospheres of white dwarfs descending from low metallicity progenitors (Miller Bertolami et al. 2013). More recently, Althaus et al. (2015) have expanded the calculations of Miller Bertolami et al. (2013) and have shown that for progenitor metallicities between 0.00003 and 0.001 , and in the absence of carbon enrichment due to the occurrence of a third dredge-up episode, the resulting hydrogen envelope of low-mass white dwarfs is thick enough to make stable hydrogen burning the most important energy source even at low luminosities. Although in some cases this may not be a relevant source of energy, it may have non-negligible effects when computing the ages of very old clusters of low metallicity.

In this paper we analyze the white dwarf population of the old, metal-poor globular cluster NGC 6397. We use an advanced population synthesis code based on Monte Carlo techniques, which incorporates the most recent and reliable cooling sequences, a modern description of the main properties of NGC 6397, and an accurate modeling of the observational biases. Our paper is organized as follows. In Sects. 2 and 3 we describe our population synthesis code and the observed sample. Section 4 is devoted to analyzing the results of our simulations. Specifically, we study the role of residual hydrogen burning, the effects of the slope of the adopted initial mass function, the possible effects of mass segregation on the white dwarf population, and the fraction of white dwarfs with hydrogen-poor atmospheres. This is based on a detailed statistical analysis of the luminosity function, of the color distribution, and of the colormagnitude diagram of the white dwarf population of NGC 6397. Finally, in Sect. 5 our main results are summarized and our conclusions are drawn.

\section{Population synthesis code}

Our synthetic population code is based on Monte Carlo techniques and has been extensively used for studying the disk (García-Berro et al. 1999; Torres et al. 2001) and halo (Torres et al. 2002; García-Berro et al. 2004) populations of single white dwarfs. An improved version of this population synthesis code has recently been employed to model the properties of the disk population of white dwarf plus main-sequence binary stars (Camacho et al. 2014). Finally, and most relevant for this study, our code has also been successfully used to model Galactic open clusters, such as NGC 6791 (García-Berro et al. 2010, 2011b), and globular clusters, such as 47 Tuc (García-Berro et al. 2014). Detailed descriptions of our Monte Carlo simulator can be found in these papers. Thus, in this section we only summarize its most salient features and refer to these works for more details.

Synthetic main-sequence stars are randomly drawn according to a Salpeter-like initial mass function. In this work we used the so-called universal mass function of Kroupa (2001). For the mass range relevant to our study, this initial mass function is totally equivalent to a two-branch power law with exponent $-\alpha$, with $\alpha=1.3$ for $0.08 \leq M / M_{\odot}<0.5$ and $\alpha=2.30$ for $M / M_{\odot} \geq 0.5$. However, for the sake of completeness and simplicity, we also use a classical Salpeter-like (Salpeter 1955) initial mass function in Sect. 4.3. This mass function is a power law with just one index, which we consider a free parameter to fit the observations. The selected range of masses at the zero-age mainsequence is that necessary to produce white dwarf progenitors of suitable masses for NGC 6397. In particular, a lower limit of $M>0.5 M_{\odot}$ guarantees that enough white dwarfs are produced for a broad range of cluster ages. Within this mass range, both initial mass functions are totally equivalent when the standard value of $\alpha=2.35$ is adopted.

We adopted an age of the cluster, $T_{\mathrm{c}}$, that falls within an interval from 11.0 to $14.5 \mathrm{Gyr}$. This was done to account for the widest relevant range of age estimates of NGC 6397. We also 
employed a star formation rate consisting of a burst of duration $\Delta t$. The width of the burst covers a sufficiently wide range of values, from 0.0 to $4.0 \mathrm{Gyr}$, to ensure that all possibilities are explored. Finally, we adopted a maximum fraction of unresolved double-degenerate binaries $f_{\mathrm{BIN}} \leq 8 \%$. This upper limit is consistent with the expected values reported by Hansen et al. (2007) and Davis et al. (2008). The masses of the individual components of the binary system were randomly drawn according to a flat distribution. However, the results are not sensitive to the choice of the mass distribution. We also considered that half of these binary systems are composed of a helium-core and a carbonoxygen white dwarf, whereas for the rest of the systems the two components are typical carbon-oxygen white dwarfs. For white dwarfs with helium cores we adopted a mean mass of $0.4 M_{\odot}$ with a Gaussian deviation of $0.15 M_{\odot}$

Once we knew which stars had time to evolve to white dwarfs, we interpolated their photometric properties using a set of theoretical cooling sequences (Miller Bertolami et al. 2013; Althaus et al. 2015) for white dwarfs with hydrogenrich atmospheres, in accordance with the observed spectroscopic classification of the white dwarfs of the cluster. This set of evolutionary sequences consists of a grid of cooling tracks for carbonoxygen white dwarfs derived from evolutionary calculations of progenitors with metallicities ranging from $Z=1.0 \times 10^{-4}$ to $Z=5.0 \times 10^{-4}$. We note here that these evolutionary sequences were evolved self-consistently from the zero-age mainsequence, through the giant phase, the thermally pulsing asymptotic giant branch (AGB) and mass-loss phases, and ultimately to the white dwarf stage. We also emphasize that these evolutionary sequences provide a self-consistent initial-to-final mass relation. For more massive oxygen-neon white dwarfs we employed the cooling sequences of Althaus et al. (2005, 2007). For heliumcore white dwarfs we used the cooling sequences of Serenelli et al. (2001), while for non-DA white dwarfs we used the cooling tracks of Bergeron et al. (2011) and the colors of Sirianni et al. (2005). An important detail here is that for consistency, the masses of the resulting synthetic white dwarfs were interpolated using the initial-to-final mass relationships obtained from the previously mentioned set of full evolutionary sequences for metal poor progenitors. Nevertheless, for the sake of completeness, we also ran some simulations for which the semi-empirical initial-to-final mass relationship of field white dwarfs (Catalán et al. 2008a,b) was used, obtaining essentially the same results.

Photometric errors were assigned randomly according to the observed distribution. Specifically, for each synthetic white dwarf the photometric errors were drawn within a hyperbolically increasing band limited by $\sigma_{1}=0.2\left(m_{\mathrm{F} 814 \mathrm{~W}}-31.0\right)^{-2}$ and $\sigma_{\mathrm{u}}-0.06=1.7\left(m_{\mathrm{F} 814 \mathrm{~W}}-31.0\right)^{-2}$, which fits the observations of Hansen et al. (2007) for the $m_{\mathrm{F} 814 \mathrm{~W}}$ magnitude well. Similar expressions were employed for the remaining magnitudes.

\section{Observed data set}

The observed data to which we compare our population synthesis models was obtained from Hansen et al. (2007) and consists of a series of images taken with the Advanced Camera for Surveys (ACS) field located $5^{\prime}$ southeast of the cluster core. Zero points for the instrumental magnitudes are 32.414 for $m_{\mathrm{F} 814 \mathrm{~W}}$ and 33.321 for $m_{\mathrm{F} 606 \mathrm{~W}}$. In addition, an absolute sharp parameter smaller than 0.02 and a round parameter smaller than 0.02 was used to remove extended sources. In Fig. 1 we show the raw color-magnitude diagram of the sample. Objects with $m_{\mathrm{F} 814 \mathrm{~W}}<2.3\left(m_{\mathrm{F} 606 \mathrm{~W}}-m_{\mathrm{F} 814 \mathrm{~W}}\right)+22.5$ are far below the main

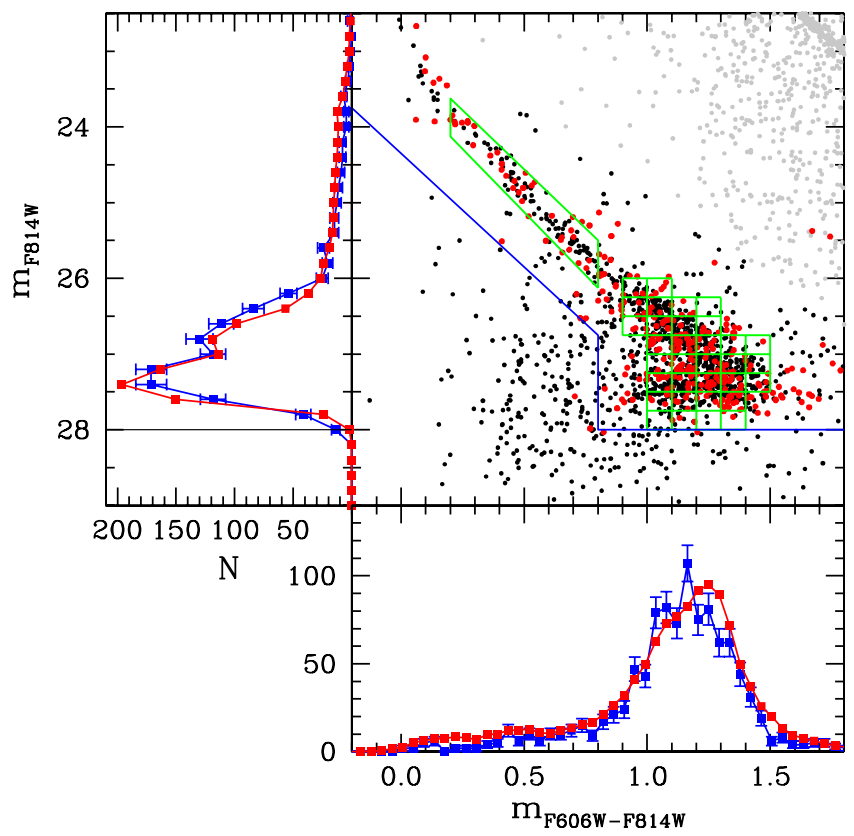

Fig. 1. White dwarf luminosity function, color-magnitude diagram, and color distribution of NGC 6397 for our best-fit model. Gray dots represent observed main-sequence stars, black dots correspond to white dwarfs, while red points denote the results of our simulations. The green squares represent the regions of the color-magnitude diagram for which we peformed a $\chi^{2}$ test, while the blue thin lines correspond to the cuts adopted to compute the distributions. The red curves correspond to the simulated distributions, while the blue curves are the observed distributions computed using our cuts. See the online edition of the journal for a color version of this figure and the main text for additional details.

sequence and can safely be considered as candidate white dwarfs (black dots in the top right panel of Fig. 1). For magnitude 27, the completeness of the sample is $80 \%$, and for higher magnitudes, this value steadily decreases, reaching $50 \%$ at magnitude 28 . The black line in the top left panel of this figure represents this limit.

A third epoch set of observations to derive deeper proper motions, which are especially useful for distinguishing white dwarfs with faint magnitudes, has not yet been published. Consequently, and to avoid contamination from galaxies, we applied a set of color cuts (represented by the blue lines in the color-magnitude diagram of Fig. 1). Objects below the blue line of Fig. 1 are not considered in our analysis. Finally, we built a clean luminosity function and a color distribution function - left and bottom panels of Fig. 1, respectively - taking into account these additional color cuts (blue lines).

\section{Results}

\subsection{Color-magnitude diagram}

We first discuss the overall shape of the color-magnitude diagram and the corresponding distributions of magnitudes and colors. All this information is displayed in Fig. 1 for a model in which we adopted an age $T_{\mathrm{c}}=12.8 \mathrm{Gyr}$, a burst duration $\Delta t=1.0 \mathrm{Gyr}$, and a fraction of binaries $f_{\mathrm{BIN}}=4 \%$, which we consider our reference model. The top right panel of this figure shows the observed stars and the synthetic white dwarfs. The results of our synthetic population calculations are represented using red dots in the color-magnitude diagram, whereas the corresponding magnitude and color distributions are shown 
Table 1. $\chi^{2}$ test of the luminosity function, color distribution, and color-magnitude diagram for different ages, durations of the star formation burst, and binary fractions.

\begin{tabular}{|c|c|c|c|c|c|c|c|c|c|}
\hline \multirow{3}{*}{$\frac{\chi^{2} \text { test }}{\Delta t(\mathrm{Gyr})}$} & \multicolumn{3}{|c|}{$\chi_{\mathrm{F} 814 \mathrm{~W}, v}^{2} / \chi_{\min }^{2}$} & \multirow{2}{*}{\multicolumn{3}{|c|}{$\frac{\chi_{\mathrm{F} 606 \mathrm{~W}-\mathrm{F} 814 \mathrm{~W}, v}^{2} / \chi_{\mathrm{min}}^{2}}{T_{\mathrm{c}}=11.0(\mathrm{Gyr})}$}} & \multicolumn{3}{|c|}{$\chi_{\mathrm{N}, v}^{2} / \chi_{\min }^{2}$} \\
\hline & \multirow[b]{2}{*}{1.0} & \multirow[b]{2}{*}{2.0} & \multirow[b]{2}{*}{3.0} & & & & \multirow[b]{2}{*}{1.0} & \multirow[b]{2}{*}{2.0} & \multirow[b]{2}{*}{3.0} \\
\hline & & & & \multicolumn{3}{|c|}{$T_{\mathrm{c}}=11.0(\mathrm{Gyr})$} & & & \\
\hline$f_{\mathrm{BIN}}=0.00$ & 9.42 & 11.31 & 12.68 & 2.69 & 3.60 & 4.67 & 4.17 & 5.33 & 5.88 \\
\hline$f_{\mathrm{BIN}}=0.02$ & 9.72 & 11.48 & 12.79 & 2.62 & 3.54 & 4.47 & 4.18 & 5.29 & 5.90 \\
\hline$f_{\mathrm{BIN}}=0.04$ & 9.71 & 11.38 & 12.86 & 2.65 & 3.56 & 4.63 & 4.16 & 5.31 & 5.97 \\
\hline \multirow[t]{2}{*}{$f_{\mathrm{BIN}}=0.06$} & 9.62 & 11.53 & 12.84 & 2.58 & 3.54 & 4.49 & 4.16 & 5.36 & 5.82 \\
\hline & \multicolumn{9}{|c|}{$T_{\mathrm{c}}=12.0(\mathrm{Gyr})$} \\
\hline$\Delta t(\mathrm{Gyr})$ & 1.0 & 2.0 & 3.0 & 1.0 & 2.0 & 3.0 & 1.0 & 2.0 & 3.0 \\
\hline$f_{\mathrm{BIN}}=0.00$ & 4.43 & 5.92 & 7.33 & 1.24 & 1.68 & 2.40 & 1.71 & 2.50 & 3.28 \\
\hline$f_{\mathrm{BIN}}=0.02$ & 4.49 & 5.95 & 7.54 & 1.28 & 1.62 & 2.37 & 1.78 & 2.44 & 3.23 \\
\hline$f_{\mathrm{BIN}}=0.04$ & 4.44 & 6.02 & 7.52 & 1.26 & 1.67 & 2.36 & 1.72 & 2.41 & 3.20 \\
\hline \multirow[t]{2}{*}{$f_{\mathrm{BIN}}=0.06$} & 4.38 & 6.07 & 7.39 & 1.30 & 1.63 & 2.31 & 1.71 & 2.45 & 3.13 \\
\hline & \multicolumn{9}{|c|}{$T_{\mathrm{c}}=13.0(\mathrm{Gyr})$} \\
\hline$\Delta t(\mathrm{Gyr})$ & 1.0 & 2.0 & 3.0 & 1.0 & 2.0 & 3.0 & 1.0 & 2.0 & 3.0 \\
\hline$f_{\mathrm{BIN}}=0.00$ & 1.95 & 2.11 & 2.86 & 1.24 & 1.08 & 1.16 & 1.43 & 1.17 & 1.48 \\
\hline$f_{\mathrm{BIN}}=0.02$ & 2.05 & 2.14 & 2.92 & 1.34 & 1.07 & 1.16 & 1.42 & 1.10 & 1.45 \\
\hline$f_{\mathrm{BIN}}=0.04$ & 1.90 & 2.09 & 2.94 & 1.32 & 1.08 & 1.15 & 1.38 & 1.12 & 1.41 \\
\hline$f_{\mathrm{BIN}}=0.06$ & 1.87 & 2.07 & 2.97 & 1.34 & 1.11 & 1.17 & 1.37 & 1.13 & 1.40 \\
\hline \multirow{6}{*}{$\begin{array}{l}\Delta t(\mathrm{Gyr}) \\
f_{\mathrm{BIN}}=0.00 \\
f_{\mathrm{BIN}}=0.02 \\
f_{\mathrm{BIN}}=0.04 \\
f_{\mathrm{BIN}}=0.06\end{array}$} & \multicolumn{9}{|c|}{$T_{\mathrm{c}}=14.0(\mathrm{Gyr})$} \\
\hline & 1.0 & 2.0 & 3.0 & 1.0 & 2.0 & 3.0 & 1.0 & 2.0 & 3.0 \\
\hline & 4.00 & 2.04 & 1.18 & 2.40 & 1.75 & 1.29 & 2.59 & 1.93 & 1.34 \\
\hline & 3.81 & 2.10 & 1.11 & 2.52 & 1.82 & 1.28 & 2.60 & 1.84 & 1.39 \\
\hline & 3.72 & 1.90 & 1.08 & 2.46 & 1.78 & 1.30 & 2.61 & 1.81 & 1.28 \\
\hline & 3.61 & 1.82 & 1.07 & 2.52 & 1.79 & 1.33 & 2.54 & 1.86 & 1.38 \\
\hline
\end{tabular}

Notes. We list the reduced and normalized value of $\chi^{2}$, that is the value of $\chi_{v}^{2}$ over its minimum value.

as red lines in Fig. 1. To obtain the color-magnitude diagram shown in this figure, we fit the position of the bright branch of the degenerate cooling sequence, obtaining an apparent distance modulus $(m-M)_{\mathrm{F} 814 \mathrm{~W}}=12.42_{-0.09}^{+0.05}$ and a color excess $E(\mathrm{~F} 606 \mathrm{~W}-\mathrm{F} 814 \mathrm{~W})=0.22 \pm 0.02$. These values agree with those of Richer et al. (2013) and references therein.

Our reference model and the observations obviously agree excellently well. However, in our modeling we went one step beyond this and performed a $\chi^{2}$ analysis using the following strategy. We computed independent $\chi^{2}$ tests for the magnitude $\left(\chi_{\mathrm{F} 814 \mathrm{~W}}^{2}\right)$ and color $\left(\chi_{\mathrm{F} 606 \mathrm{~W}-\mathrm{F} 814 \mathrm{~W}}^{2}\right)$ distributions. Additionally, we calculated the number of white dwarfs inside each of the green boxes in the color-magnitude diagram of Fig. 1 - which are the same regions of this diagram used by Hansen et al. (2013) to compare observations and simulations - and we performed an additional $\chi^{2}$ test, $\chi_{N}^{2}$. Then, we obtained for each of the three independent $\chi^{2}$ tests the reduced value of $\chi^{2}, \chi_{v}^{2}=\chi^{2} / v$, where $v$ is the number of degrees of freedom, $v=N-p-1$, where $N$ is the number of bins and $p$ the number of free parameters. Then the reduced $\chi^{2}$ values were normalized to the lowest value for each of the tests and added in quadrature. Employing this procedure, only one estimator must be minimized to find the model that best fits all distributions. Clearly, this strategy is equivalent to a maximum likelihood method, hence the results of both approaches are consistently similar. For illustrative purposes, we list a representative set of values for the different tests and free parameters studied here in Table 1 . It is worth mentioning here that although we used a $\chi^{2}$ test, our final aim is to estimate the values of the free parameters that best fit the observed data instead of obtaining an absolute probability of agreement of our models with the observed data.

In Fig. 2 we show the probability distribution for different pairs of the studied free parameters using a gray scale - the cluster age, $T_{\mathrm{c}}$, the burst duration, $\Delta t$, and the binary fraction, $f_{\mathrm{BIN}}$. Also shown, using red lines, are the curves enclosing the regions of $68 \%, 90 \%$, and $95 \%$ confidence level. Finally, our bestfit model is marked with a red cross and our reference model with a white one. Using the procedure previously detailed, we found that the model that best fits the observed data has the following properties. The age of the cluster is $T_{\mathrm{c}}=13.9 \mathrm{Gyr}$, the duration of the burst of star formation is $\Delta t=3.3 \mathrm{Gyr}$ and the binary fraction of the cluster is $f_{\mathrm{BIN}}=4 \%$. One point of concern regarding our best-fit model is its very great age spread, 3.3 Gyr. This is unusual in most globular cluster except for two, $\omega$ Centauri - see Villanova et al. (2014) for a recent discussion about this issue - and M 54 (Siegel et al. 2007). Nevertheless, we remark that previous theoretical efforts of modeling the white dwarf luminosity function of NGC 6397 could only achieve satisfactory fits when a constant star formation rate during the entire life of the cluster was employed (Winget et al. 2009). However, we judge that the burst duration obtained using this procedure is totally unrealistic, and that our reference model, for which we adopted a typical burst duration of $1.0 \mathrm{Gyr}$ is more realistic. We elaborate on the reasons for this below.

Inspection of the top and bottom panels of Fig. 2 reveals that there is no correlation between the adopted fraction of binaries and the duration of the burst or the cluster age. Accordingly, $f_{\mathrm{BIN}}$ can be considered as an independent free parameter. Thus, its value has no major effects on the final results. In contrast, the middle panel of Fig. 2 unveils a clear unphysical correlation between the duration of the burst and the age of the cluster. Ages shorter than that of our best-fit model are possible provided that lower values of the burst duration are adopted, and the reverse is also true. Ages as short as $12.8 \mathrm{Gyr}$ are compatible, at the $95 \%$ confidence level, with the observations if the initial burst of star formation lasted for 1.0 Gyr. Even a cluster age of $12.4 \mathrm{Gyr}$ is compatible at the $90 \%$ confidence level with the observed data if 

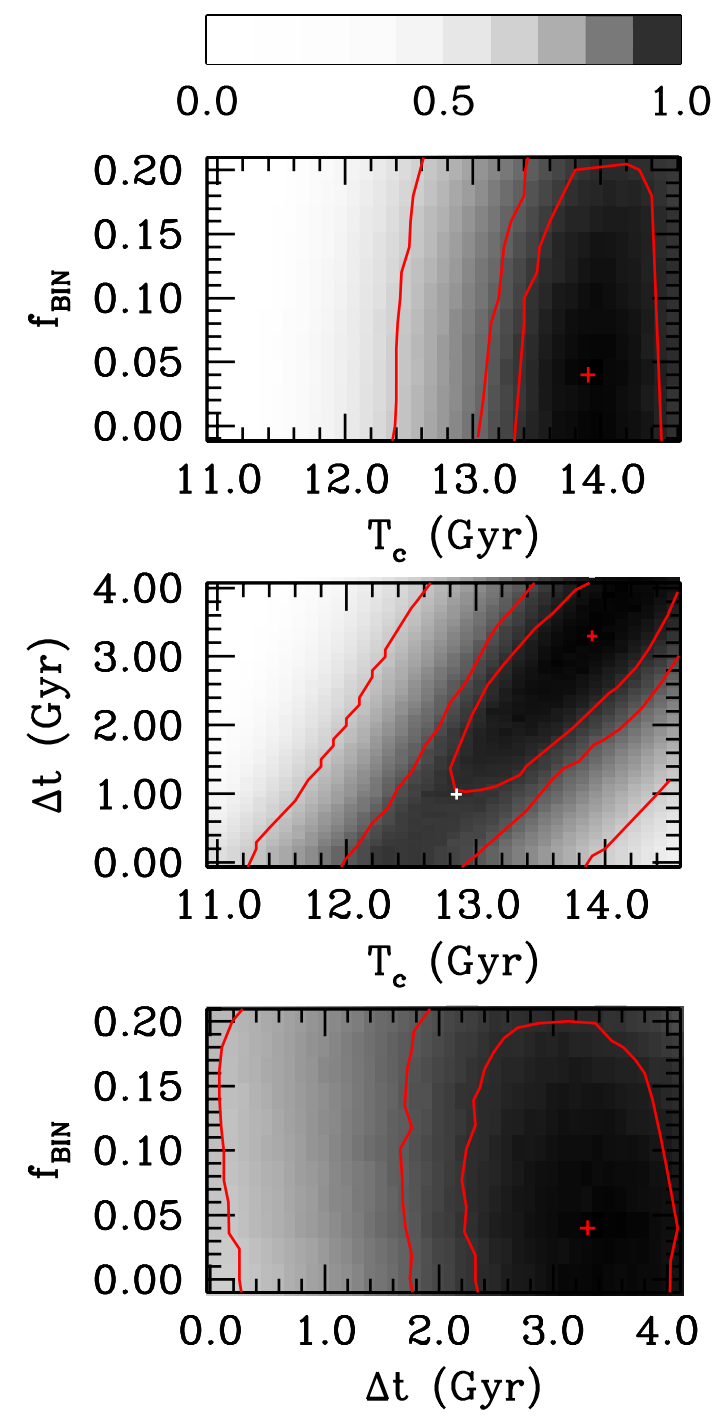

Fig. 2. Probability density distribution for different pairs of free parameters (gray scale). Marked with a cross are the best-fit values, while the red lines represent the $68 \%, 90 \%$, and $95 \%$ confidence levels. The white cross corresponds to our reference model.

the duration of the burst of star formation is 0.1 Gyr. Moreover, if $\Delta t=0.0 \mathrm{Gyr}$ is adopted, the age of the cluster is $12.34_{-0.4}^{+0.5} \mathrm{Gyr}$, in agreement with other ages estimates (Hansen et al. 2013). All this is due to the very flat maximum of the probability distributions displayed in Fig. 2.

Since this is an important point, we explored the origin of this unphysical correlation between the age of the cluster and the duration of the episode of star formation computed using the available white dwarf luminosity function. We found that the main reason is that the observed stars with $m_{\mathrm{F} 814 \mathrm{~W}} \geq 26$ have large photometric errors. Thus, the precise position of the maximum of the observed white dwarf luminosity function cannot be determined with good accuracy. Hence, age determinations relying on the observed luminosity function are heavily influenced by photometric errors. Additionally, such an extended episode of star formation would influence the morphology of the colormagnitude diagram of main-sequence stars of NGC 6397. With the high-accuracy photometric data collected with the Hubble Space Telescope today (Milone et al. 2012), this age spread should be detectable, and this is not the case. In conclusion, we judge that the adopted photometric dataset is not yet appropriate for this kind of analysis, and that there are solid reasons to suspect that the duration of the initial burst of star formation is shorter than that obtained from our analysis. Consequently, from now on we stick to our reference model, in which a burst duration of 1.0 Gyr is adopted.

Finally, we checked the sensitivity of our results to the distance modulus and the reddening. Specifically, we varied the reddening by \pm 0.02 , the nominal errors in its determination, and we found that the derived age for the cluster when our reference model is adopted varies by \pm 0.02 Gyr. We repeated the same procedure for the value of the distance modulus and found that in this case the age of the cluster varies by \pm 0.3 Gyr. Furthermore, fixing the duration of the initial burst of star formation, we obtained a conservative estimate of the error associated with the derived age of the cluster, and also to the fraction of binaries. We obtained $\sigma_{T_{\mathrm{c}}}={ }_{-0.75}^{+0.50} \mathrm{Gyr}$, and $\sigma_{\mathrm{fBIN}_{\mathrm{BI}}}{ }_{-0.04}^{+0.14}$. As a final analysis, we also studied the robustness of our results to the completeness of the observed sample. In particular, we discarded $20 \%$ of synthetic white dwarfs with magnitudes smaller than 26 , while for magnitudes between 26 and 28 we assumed that the completeness decreases linearly until $50 \%$, and removed the corresponding synthetic white dwarfs. We then computed the white dwarf luminosity function and color distribution of the resulting sample and found that the results were essentially the same. We thus conclude that the age derived for our reference model is robust.

\subsection{Role of residual nuclear burning}

As mentioned earlier, residual nuclear burning in white dwarf atmospheres can be an important source of energy, especially for white dwarfs descending from progenitors of low metallicity. This is so because in the absence of third dredge-up, low-metallicity progenitors depart from the AGB with thicker envelopes (Iben \& MacDonald 1986), thus resulting in white dwarfs with thicker hydrogen envelopes. Consequently, residual hydrogen burning is expected to become relevant in white dwarfs with low-metallicity progenitors. In particular, Renedo et al. (2010) found that in white dwarfs resulting from progenitors with subsolar metallicity, residual hydrogen burning may account for about $30 \%$ of the luminosity when cooling has proceeded down to luminosities ranging from $L \sim 10^{-2} L_{\odot}$ to $10^{-3} L_{\odot}$. This contribution is even more relevant when very low metallicity progenitors $(Z=0.0001)$ and white dwarf masses lower than $\sim 0.6 M_{\odot}$ are considered. In these cases, nuclear reactions are the main contributor to the stellar luminosity for luminosities as $\operatorname{low}$ as $\log \left(L / L_{\odot}\right) \simeq-3.2$ (Miller Bertolami et al. 2013). For instance, consider an otherwise typical white dwarf with $M_{\mathrm{WD}}=0.6 M_{\odot}$, descending from a progenitor with the metallicity of NGC 6397. It takes about 1.04 Gyr for this white dwarf to cool down to $\log \left(L / L_{\odot}\right) \simeq-3.2$ when no nuclear burning is considered, while when residual nuclear burning is considered, the cooling age is $\sim 1.49 \mathrm{Gyr}$. Although these differences become smaller for fainter luminosities, residual hydrogen burning might still play a significant role in shaping the white dwarf luminosity function at moderately low luminosities. Given the distance modulus of NGC 6397, these luminosities correspond to apparent magnitudes of about $m_{\mathrm{F} 814 \mathrm{~W}} \approx 24 \sim 26$, in the hot branch of the white dwarf luminosity function.

To assess the possible effect of residual nuclear burning, we used a second set of cooling sequences where this source of energy was disregarded. As before, we built a grid of sequences for different masses and ages and interpolated for the precise metallicity of NGC 6397, keeping the duration of the initial burst of star formation constant, for which we adopted, as previously 


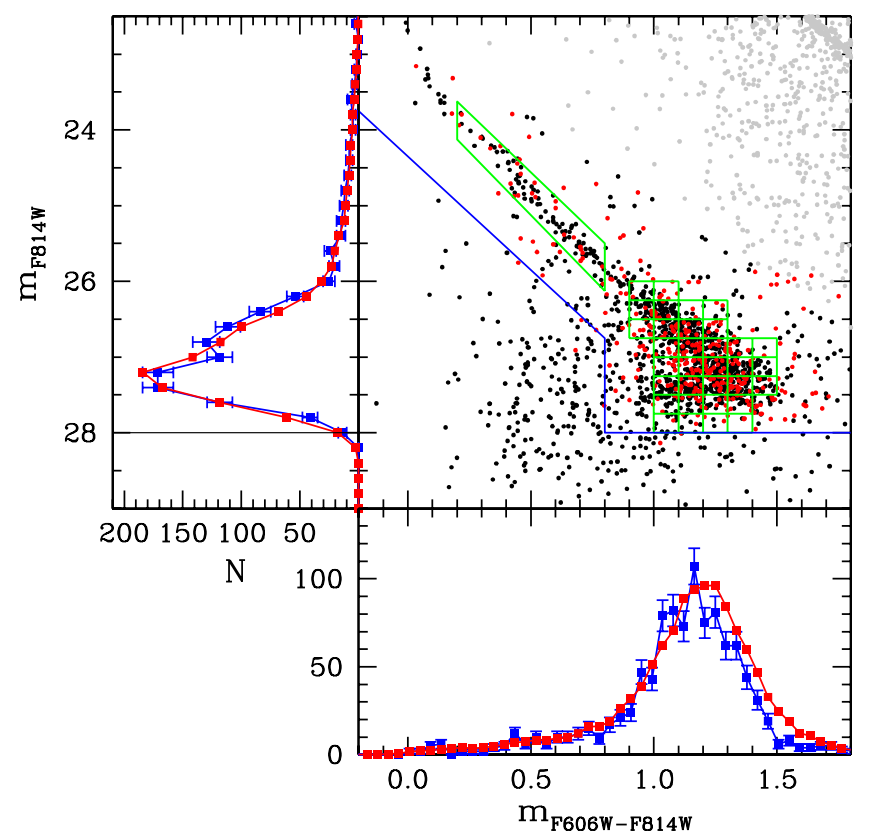

Fig. 3. Same as Fig. 1, but for the model with no residual hydrogen burning, see Sect. 4.2.

discussed, $\Delta t=1.0$ Gyr. Then we ran our Monte Carlo simulator for the full range of the free parameters and obtained the combined $\chi^{2}$ for each simulation, employing the procedure explained in Sect. 4.1. The white dwarf luminosity function, the color-magnitude diagram, and the color distribution for the bestfit model is shown in Fig. 3. Although the general agreement between the simulated and observed samples for the different distributions of Fig. 3 is again quite good, the fit of some features of the observed distributions is worse when compared with that obtained when residual nuclear burning is taken into account. For instance, as can be seen in Figs. 1 and 3, the small, secondary peak of the observed white dwarf luminosity function at $m_{\mathrm{F} 814 \mathrm{~W}} \approx 26.75$ is best fit when the sequences including residual hydrogen burning are considered. Moreover, when no residual nuclar burning is considered, an excess of simulated white dwarfs redder than $m_{\mathrm{F} 606 \mathrm{~W}-\mathrm{F} 814 \mathrm{~W}} \approx 1.2$ is quite apparent in the bottom panel of Fig. 3. According to these findings, for the rest the calculations presented in this work we only employ evolutionary sequences in which residual hydrogen burning in white dwarf atmospheres is considered.

\subsection{Initial mass function}

The initial mass function is a key ingredient in modeling the observed properties of globular clusters. The most commonly used initial mass function consists of a power law with a characteristic power index $\alpha, \Phi(M) \propto M^{-\alpha}$, where $M$ is the mass at the zero-age main sequence. It is generally accepted that the value of the power -law index should not differ much from $\alpha=2.35$ (Salpeter 1955). However, it has recently been suggested that the initial mass function of NGC 6397 could have a flatter slope see (Richer et al. 2008) and references therein. In this section we explore this possibility.

As previously, we consider the model described in Sect. 4.1, but now we consider $\alpha$ to be a free parameter of the fit, we allow it to vary between 1.35 and 3.35, and as we did before we computed the corresponding combined value of $\chi^{2}$. In Fig. 4 we show the result of this procedure. The best-fit model corresponds

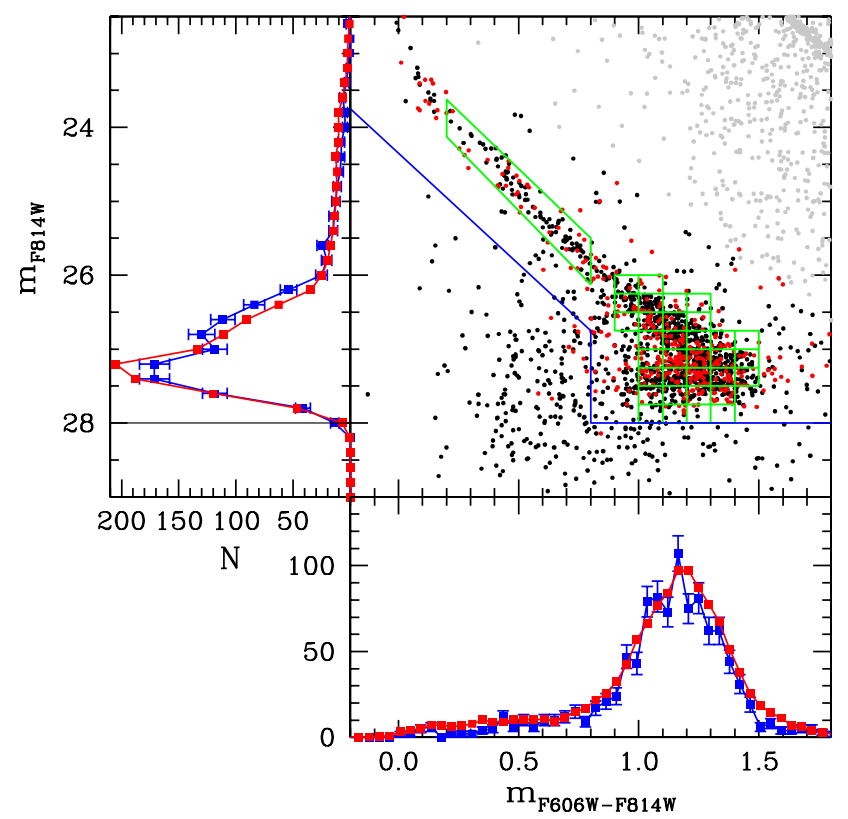

Fig. 4. Same as Fig. 1, but for the model in which the slope of the initial mass function is allowed to vary (see Sect. 4.3 for details).

to $\alpha=1.95$. The remaining values of the free parameters for this best-fit model are similar to that obtained when a standard Salpeter-like initial mass function with $\alpha=2.35$ is adopted see Sect. 4.1. Nevertheless, including $\alpha$ as a new free parameter does not substantially improve the fit. Therefore, we conclude that unless a more restrictive observational data set is employed, a standard value of $\alpha$ adequately describes the global observed properties of the white dwarf population of NGC 6397.

\subsection{Fraction of non-DA white dwarfs}

Although observations indicate that the fraction of non-DA white dwarfs in NGC 6397 is small, on the order of $f_{\text {non-DA }} \sim$ 4\% (Hansen et al. 2007; Strickler et al. 2009), its effects on the color-magnitude diagram and on the corresponding white dwarf luminosity function and color distribution are interesting to explore. To this end, we show in Fig. 5 the results for an unrealistic fraction of $20 \%$ of non-DA white dwarfs. In this colormagnitude diagram, non-DA white dwarfs are shown as blue dots. When fractions smaller than $20 \%$ are used, very few nonDA white dwarfs are found. Thus, this figure should be regarded as an extreme case to illustrate the effects of varying the fraction of non-DA white dwarfs.

As previously, we considered the model described in Sect. 4.1, but we now considered $f_{\text {non-DA }}$ to be a free parameter of the fit and computed the corresponding combined value of $\chi^{2}$, now keeping now the fraction of unresolved binaries fixed at 0.04 , the best-fit value obtained previously. The value of the fraction of non-DA white dwarfs that best fits the observed data is $f_{\text {non-DA }}=0$. Hence, our analysis concurs with observations that NGC 6397 has a negligible fraction of non-DA white dwarfs. However, we note that small fractions of non-DA white dwarfs are allowed by the present set of observed data at the $95 \%$ confidence level.

\subsection{Mass segregation in NGC 6397}

Another important problem worth investigating is the dynamical evolution of NGC 6397. The dynamical evolution of globular 


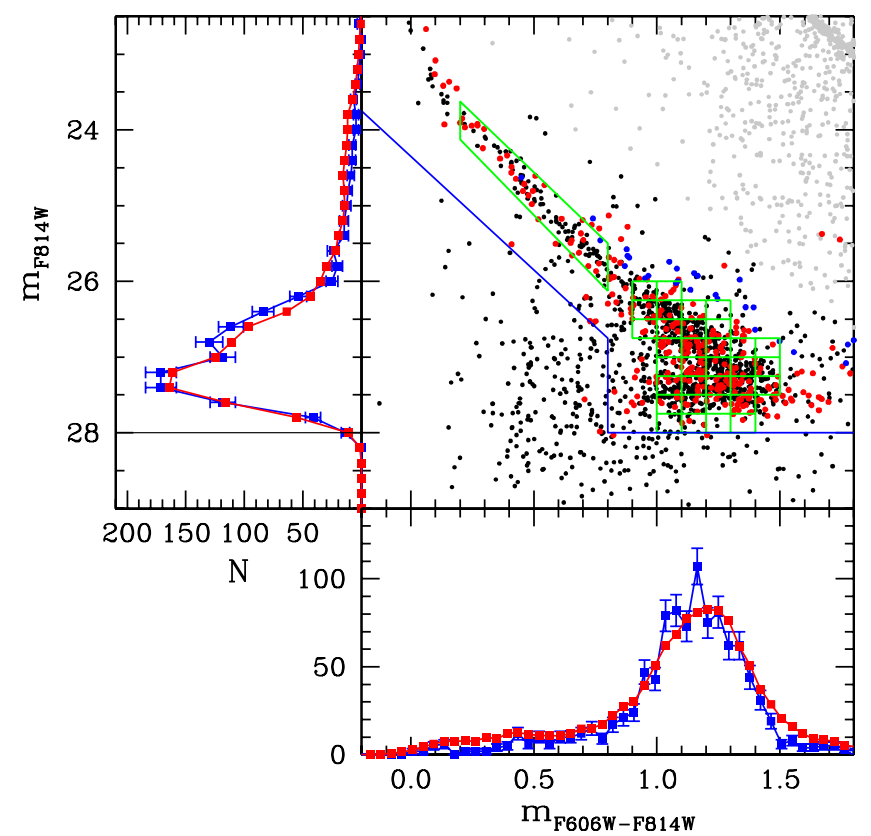

Fig. 5. Same as Fig. 1, but for a model in which we adopt a fraction of non-DA white dwarfs of $20 \%$ (see Sect. 4.4 for details).

clusters is primarily governed by the gravitational attraction between individual stars, although the interplay of the evolution of their individual stars and binary encounters also plays an important role. The high stellar densities in the cores of globular clusters favor binary encounters. In these dynamical interactions the more massive star usually transfers kinetic energy to the less massive one. Eventually, high-mass stars clump in the core of the cluster, while low-mass stars move to the outskirts of it or even escape the cluster.

Given that the observed sample of white dwarfs employed in our study was selected from the ACS field, which is located $5^{\prime} \mathrm{SE}$ of the cluster center, it is reasonable to expect that mass segregation may play a role. Moreover, the evaporation time of NGC 6397 is 3 Gyr (Heyl et al. 2012), implying that an important fraction of stars, in particular those with low masses, could have escaped from the cluster. However, the existence of mass segregation in NGC 6397 is still controversial - see, for instance, the recent paper of Martinazzi et al. (2014) and references therein - and a study employing white dwarf stars would help in settling this question. Although an exhaustive assessment of the dynamical evolution of NGC 6397 using the properties of the white dwarf population is beyond the scope of the present paper, a simple approach is worth pursuing, since a comparison of the properties of the simulated population with the observed sample could shed some light on the existence of mass segregation.

To test how mass segregation affects the white dwarf population of NGC 6397, we proceeded as follows. The bottom panel of Fig. 6 displays the distribution of white dwarf masses as a function of the magnitude for our synthetic population. Black points denote typical carbon-oxygen white dwarfs, whereas the gray points represent white dwarfs with masses higher than a variable threshold $M_{\mathrm{u}}$ (upper red line) and smaller than a given adjustable value $M_{1}$ (lower red line). We also show the observed white dwarf luminosity function, which is shown as a black line in the top panel of Fig. 6, while the gray line is used to represent the luminosity function of the entire synthetic sample. Finally, the white dwarf luminosity function for stars with

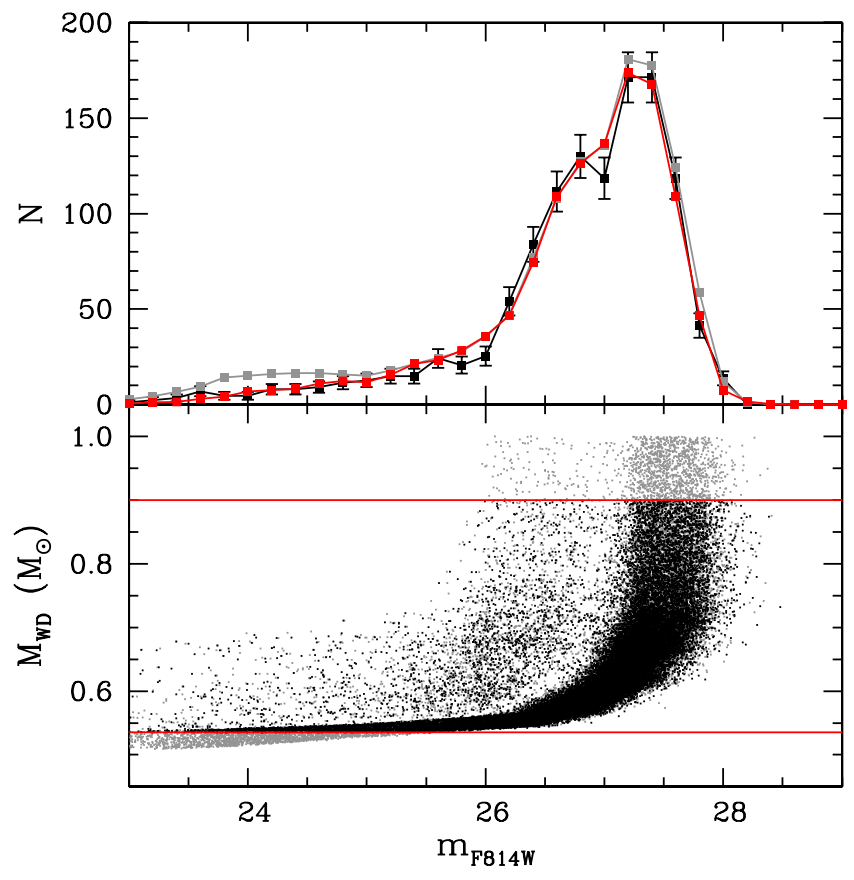

Fig. 6. Top panel: clean white dwarf luminosity function of NGC 6397 (black line) compared with the synthetic luminosity function for the entire sample of white dwarfs (gray line) and that obtained after eliminating massive and low-mass white dwarfs (red line). Bottom panel: mass distribution as a function of the magnitude for our simulated sample (black dots). The mass cuts are also displayed in this panel as horizontal red lines.

masses $M_{1}<M<M_{\mathrm{u}}$ is represented using a red line. Figure 6 shows that the bright portion $\left(m_{\mathrm{F} 814 \mathrm{~W}} \simeq 23 \sim 26\right)$ of the luminosity function is dominated by low-mass white dwarfs. By varying the lower mass cut, we fit the shape of the hot branch of the luminosity function and obtained that the value of $M_{1}$ that best fits the luminosity function is $0.535 M_{\odot}$. On the other hand, massive white dwarfs dominate the region beyond the maximum of the white dwarf luminosity function. By adjusting the value of the upper cut in masses the height of the maximum of the white dwarf luminosity function can be fit. The upper mass limit obtained in this way is $0.905 M_{\odot}$. These two values are those shown in Fig. 6 as horizontal red lines. We note that the white dwarf luminosity function obtained after applying these two mass cuts (the red line in the top panel of Fig. 6) fits the observed luminosity function extremely well. In fact, for the particular model analyzed in Fig. 6, which corresponds to a cluster age of $12.8 \mathrm{Gyr}$, a burst duration of $1.0 \mathrm{Gyr}$ and a binary fraction of $10 \%$, the value of the reduced $\chi^{2}$ is 1.20 when the mass cuts are applied. This number has to be compared with that obtained when no mass cuts are employed, which is $\chi^{2}=1.67$, indicating a much better fit. Obviously, these cuts reduce the size of the synthetic white dwarf sample. For the best-fit mass cuts, this reduction amounts to $10 \%$. Hence, we conclude that mass segregation plays a role in the fine-tuning of the luminosity function.

\section{Conclusions}

We studied the white dwarf population of NGC 6397. We employed a modern Monte Carlo population synthesis code, which incorporates the most advanced descriptions of the observational biases and selection procedures and recently obtained cooling sequences for the appropriate metallicity of this cluster. We compared the results of our simulations with the most recent 
Table 2. Age determinations for NGC 6397.

\begin{tabular}{ll}
\hline \hline$T_{\mathrm{c}}(\mathrm{Gyr})$ & Reference \\
\hline $12.0 \pm 0.8$ & Anthony-Twarog \& Twarog (2000) \\
$13.4 \pm 0.8$ & Chaboyer et al. (2001) \\
$13.9 \pm 1.1$ & Gratton et al. (2003) \\
$11.47 \pm 0.47$ & Hansen et al. (2007) \\
$12.0_{-1.0}^{+0.5}$ & Winget et al. (2009) \\
$13.50 \pm 0.50$ & Dotter et al. (2010) \\
$13.00 \pm 0.25$ & VandenBerg et al. (2013) \\
$12.8_{-0.75}^{+0.5}$ & This work \\
\hline
\end{tabular}

observational sample of the white dwarf population of this cluster, which was obtained using deep Hubble Space Telescope observations. In particular, our simulations were compared with the general appearance of the color-magnitude diagram, with the color distribution, and with the white dwarf luminosity function in a quantitative manner, obtaining probability distributions for each pair of the most important free parameters of the fit. These are the age of the cluster, the duration of the initial burst of star formation, and the fraction of unresolved binaries. We also studied other key ingredients in modeling the white dwarf population of NGC 6397. These include the adopted reddening and distance of the cluster, the role of the completeness of the observed sample, the fraction of unresolved binaries, the initial-to-final mass relation, the adopted white dwarf cooling sequences, the initial mass function, the fraction of non-DA white dwarfs, and the impact of mass segregation. Our results agree very well with the observed data and are largely independent of model assumptions. However, the probability distributions show broad, flat maxima that prevent us from simultaneously obtaining accurate determinations of the age of the cluster and of the duration of its initial star formation burst. The origin of these flat maxima ar the photometric errors of the current observed data set, which are still large.

The age of NGC 6397 derived using the white dwarf cooling sequence is $12.8_{-0.75}^{+0.50} \mathrm{Gyr}$ when the duration of the initial burst of star formation is 1.0 Gyr. The uncertainty in the age of the cluster introduced by the uncertainty in the distance modulus is $\pm 0.3 \mathrm{Gyr}$, while that introduced by the reddening is negligible when compared with the previous one. This age estimate agrees well with the age determinations obtained using the mainsequence turnoff - see Table 2. Specifically, our age determination is slightly shorter than the most recent determinations obtained using main-sequence stars. Specifically, VandenBerg et al. (2013) obtained 13.00 \pm 0.25 Gyr, and Dotter et al. (2010) derived $13.50 \pm 0.50 \mathrm{Gyr}$, somewhat different than previous estimates. For instance, Gratton et al. (2003) obtained 13.9 $\pm 1.1 \mathrm{Gyr}$ and Chaboyer et al. (2001) derived 13.4 \pm 0.8 Gyr. However, our age estimate is in line with the age derived by Anthony-Twarog \& Twarog (2000), who obtained $12.0 \pm 0.8$ Gyr. We also find that our age estimate for NGC 6397 is significantly older than those of Hansen et al. (2007), $11.47 \pm 0.47 \mathrm{Gyr}$, and Winget et al. (2009), 12.0 $0_{-1.0}^{+0.5} \mathrm{Gyr}$, which were obtained using the position of the cutoff of the white dwarf luminosity function. This discrepancy can be partially attributed to the fact that we used updated evolutionary tracks. This includes not only the white dwarf cooling sequences, but also the main-sequence lifetimes of white dwarf progenitors and the initial-final mass relation, which in our calculations were computed for the appropriate metallicity of NGC 6397. However, the use of reliable evolutionary tracks is not the only reason for this, and we stress that other factors, such as the way in which the synthetic population of white dwarfs is generated, taking into account all the known observational biases and selection procedures, are also important to obtain reliable ages.

Another interesting result of our study is that we found that the fraction of binaries is close to zero. This is particularly reassuring, as the percentage of binaries in our best-fit model $(\sim 4.0 \%)$ is very similar to that obtained for main-sequence stars - see Tables 2 and 3 in Milone et al. (2012).

After estimating the age and percentage of unresolved binaries of NGC 6397, we decided to study other important properties of the model. We first studied the role of hydrogen residual burning in the atmospheres of white dwarfs with very metal poor progenitors. To this end, we computed a complete set of full evolutionary sequences in which residual nuclear burning was (artificially) not considered. The results of this numerical experiment indicate that although good fits to the observed properties of the white dwarf population of NGC 6397 can also be obtained using this set of evolutionary tracks, the fit is better when the cooling sequences in which residual hydrogen burning is considered are included. This result sheds light on the importance of third dredge-up and extra mixing episodes in lowmass, low-metallicity AGB stars, for which theoretical and observational evidence is not conclusive about their occurrence. As shown in Althaus et al. (2015), in the absence of third dredge-up episodes during the AGB phase, most of the evolution of white dwarfs resulting from low-mass $\left(M<1.25 M_{\odot}\right)$, low-metallicity progenitors is dominated by stable hydrogen burning. In view of our result that low-mass white dwarfs in NGC 6397 are expected to sustain significant residual hydrogen burning, we conclude that their low-mass progenitors would not have suffered from the carbon-enrichment due to third dredge-up during their AGB evolution.

Another possible concern might be the slope of the initial mass function. To assess this point, we conducted a series of simulations in which we varied the power-law index of the initial mass function, and we found that that although a shallower slope will fit the properties of the observed sample slightly better, the standard value of the Salpeter mass function fits these properties equally well within a $95 \%$ confidence level. We also studied the effect of adopting a different fraction of non-DA white dwarfs and found that our best-fit model corresponds to a negligible fraction of these stars, in agreement with observations. Finally, we made a preliminary test to assess the effects of mass segregation on the white dwarf population of NGC 6397. By adjusting the bright and the dim portion of the luminosity function, we obtained that white dwarf with masses higher than $0.905 M_{\odot}$ or lower than $0.535 M_{\odot}$ are absent from the observed sample, possibly as a consequence of mass segregation. When this is taken into account in the simulated populations, the observed and theoretical data agree excellently well.

In summary, we have conducted the most complete and advanced population synthesis study of the white dwarf population of the old, metal-poor, globular cluster NGC 6397. This study allowed us toderive useful constraints on the characteristics of this cluster and to estimate its age. Nevertheless, we emphasize that a better observational data set, with smaller photometric errors for white dwarfs of magnitudes $\gtrsim 27$, will help in obtaining even more reliable estimates of its age and of its star formation history.

Acknowledgements. This research was partially supported by MCINN grant AYA2014-59084-P, by the European Union FEDER funds, by the AGAUR (Spain), by the AGENCIA through the Programa de Modernización Tecnológica BID 1728/OCAR, and by the PIP 112-200801-00940 grant from CONICET (Argentina). We also thank B. M. S. Hansen for stimulating discussions and for providing us with the observed data. 


\section{References}

Althaus, L. G., García-Berro, E., Isern, J., \& Córsico, A. H. 2005, A\&A, 441, 689

Althaus, L. G., García-Berro, E., Isern, J., Córsico, A. H., \& Rohrmann, R. D. 2007, A\&A, 465, 249

Althaus, L. G., Córsico, A. H., Isern, J., \& García-Berro, E. 2010, A\&ARv, 18, 471

Althaus, L. G., Camisassa, M. E., Miller Bertolami, M. M., Córsico, A. H., \& García-Berro, E. 2015, A\&A, 576, A9

Anthony-Twarog, B. J. \& Twarog, B. A. 2000, AJ, 120, 3111

Avila, M. L., Rogachev, G. V., Koshchiy, E., et al. 2015, Phys. Rev. Lett., 114, 071101

Bedin, L. R., Salaris, M., Piotto, G., et al. 2005, ApJ, 624, L45

Bedin, L. R., King, I. R., Anderson, J., et al. 2008a, ApJ, 678, 1279

Bedin, L. R., Salaris, M., Piotto, G., et al. 2008b, ApJ, 679, L29

Bedin, L. R., Salaris, M., Piotto, G., et al. 2009, ApJ, 697, 965

Bedin, L. R., Salaris, M., King, I. R., et al. 2010, ApJ, 708, L32

Bedin, L. R., Salaris, M., Anderson, J., et al. 2015, MNRAS, 448, 1779

Bellini, A., Bedin, L. R., Piotto, G., et al. 2010, A\&A, 513, A50

Bellini, A., Anderson, J., Salaris, M., et al. 2013, ApJ, 769, L32

Bergeron, P., Wesemael, F., Dufour, P., et al. 2011, ApJ, 737, 28

Camacho, J., Torres, S., García-Berro, E., et al. 2014, A\&A, 566, A86

Catalán, S., Isern, J., García-Berro, E., \& Ribas, I. 2008a, MNRAS, 387, 1693

Catalán, S., Isern, J., García-Berro, E., et al. 2008b, A\&A, 477, 213

Chaboyer, B., Fenton, W. H., Nelan, J. E., Patnaude, D. J., \& Simon, F. E. 2001, ApJ, 562, 521

Córsico, A. H., Althaus, L. G., Miller Bertolami, M. M., et al. 2012, MNRAS, 424, 2792

Davis, D. S., Richer, H. B., Anderson, J., et al. 2008, AJ, 135, 2155

di Criscienzo, M., D’Antona, F., \& Ventura, P. 2010, A\&A, 511, A70

Dotter, A., Sarajedini, A., Anderson, J., et al. 2010, ApJ, 708, 698

Garcia-Berro, E., Hernanz, M., Isern, J., \& Mochkovitch, R. 1988a, Nature, 333, 642

Garcia-Berro, E., Hernanz, M., Mochkovitch, R., \& Isern, J. 1988b, A\&A, 193, 141

García-Berro, E., Hernanz, M., Isern, J., \& Mochkovitch, R. 1995, MNRAS, 277,801

García-Berro, E., Torres, S., Isern, J., \& Burkert, A. 1999, MNRAS, 302, 173

García-Berro, E., Torres, S., Isern, J., \& Burkert, A. 2004, A\&A, 418, 53

García-Berro, E., Torres, S., Althaus, L. G., et al. 2010, Nature, 465, 194

García-Berro, E., Lorén-Aguilar, P., Torres, S., Althaus, L. G., \& Isern, J. 2011a,

J. Cosmol. Astropart. Phys., 5, 21
García-Berro, E., Torres, S., Renedo, I., et al. 2011b, A\&A, 533, A31

García-Berro, E., Torres, S., Althaus, L. G., \& Miller Bertolami, M. M. 2014, A\&A, 571, A56

Goldsbury, R., Heyl, J., Richer, H. B., et al. 2012, ApJ, 760, 78

Gratton, R. G., Bragaglia, A., Carretta, E., et al. 2003, A\&A, 408, 529

Hansen, B. M. S., Brewer, J., Fahlman, G. G., et al. 2002, ApJ, 574, L155

Hansen, B. M. S., Anderson, J., Brewer, J., et al. 2007, ApJ, 671, 380

Hansen, B. M. S., Kalirai, J. S., Anderson, J., et al. 2013, Nature, 500, 51

Heyl, J. S., Richer, H., Anderson, J., et al. 2012, ApJ, 761, 51

Horowitz, C. J., Schneider, A. S., \& Berry, D. K. 2010, Phys. Rev. Lett., 104, 231101

Iben, Jr., I. \& MacDonald, J. 1986, ApJ, 301, 164

Isern, J., Hernanz, M., \& Garcia-Berro, E. 1992, ApJ, 392, L23

Isern, J., Mochkovitch, R., García-Berro, E., \& Hernanz, M. 1997, ApJ, 485, 308

Isern, J., García-Berro, E., Hernanz, M., \& Chabrier, G. 2000, ApJ, 528, 397

Isern, J., García-Berro, E., Torres, S., \& Catalán, S. 2008, ApJ, 682, L109

Isern, J., García-Berro, E., Althaus, L. G., \& Córsico, A. H. 2010, A\&A, 512, A86

Kroupa, P. 2001, MNRAS, 322, 231

Martinazzi, E., Pieres, A., Kepler, S. O., et al. 2014, MNRAS, 442, 3105

Miller Bertolami, M. M., Althaus, L. G., \& García-Berro, E. 2013, ApJ, 775, L22

Milone, A. P., Marino, A. F., Piotto, G., et al. 2012, ApJ, 745, 27

Pasquini, L., Koch, A., Smiljanic, R., Bonifacio, P., \& Modigliani, A. 2014, A\&A, 563, A3

Renedo, I., Althaus, L. G., Miller Bertolami, M. M., et al. 2010, ApJ, 717, 183

Richer, H. B., Dotter, A., Hurley, J., et al. 2008, AJ, 135, 2141

Richer, H. B., Goldsbury, R., Heyl, J., et al. 2013, ApJ, 778, 104

Salaris, M., Domínguez, I., García-Berro, E., et al. 1997, ApJ, 486, 413

Salaris, M., Althaus, L. G., \& García-Berro, E. 2013, A\&A, 555, A96

Salpeter, E. E. 1955, ApJ, 121, 161

Serenelli, A. M., Althaus, L. G., Rohrmann, R. D., \& Benvenuto, O. G. 2001, MNRAS, 325, 607

Siegel, M. H., Dotter, A., Majewski, S. R., et al. 2007, ApJ, 667, L57

Sirianni, M., Jee, M. J., Benítez, N., et al. 2005, PASP, 117, 1049

Strickler, R. R., Cool, A. M., Anderson, J., et al. 2009, ApJ, 699, 40

Torres, S., García-Berro, E., Burkert, A., \& Isern, J. 2001, MNRAS, 328, 492

Torres, S., García-Berro, E., Burkert, A., \& Isern, J. 2002, MNRAS, 336, 971

VandenBerg, D. A., Brogaard, K., Leaman, R., \& Casagrande, L. 2013, ApJ, 775,134

Villanova, S., Geisler, D., Gratton, R. G., \& Cassisi, S. 2014, ApJ, 791, 107

Winget, D. E., Kepler, S. O., Campos, F., et al. 2009, ApJ, 693, L6 